\title{
Charged local defects in extended systems
}

\author{
Peter A. Schultz \\ Sandia National Laboratories, Albuquerque, NM 87185 \\ ( Received:
}

The conventional approach to treating charged defects in extended systems in first principles calculations is via the supercell approximation using a neutralizing jellium background charge. I explicitly demonstrate shortcomings of this standard approach and discuss the consequences. Errors in the electrostatic potential surface over the volume of a supercell are shown to be comparable to a band gap energy in semiconductor materials, for cell sizes typically used in first principles simulations. I present an alternate method for eliminating the divergence of the Coulomb potential in supercell calculations of charged defects in extended systems that embodies a correct treatment of the electrostatic potential in the local vicinity of the a charged defect, via a mixed boundary condition approach. I present results of first principles calculations of charged vacancies in $\mathrm{NaCl}$ that illustrate the importance of polarization effects once an accurate representation of the local potential is obtained. These polarization effects, poorly captured in small supercells, also impact the energetics on the scale of typical band gap energies. 


\section{DISCLAIMER}

Portions of this document may be illegible in electronic image products. Images are produced from the best available original document. 
Density functional theory 1,2 (DFT) has proven to be highly accurate and broadly successful method for computing the structural and electronic properties of extended systems. Density functional calculations dependably produce lattice parameters and bulk moduli, for example, of crystalline bulk systems to within a few percent. These methods, particularly those using pseudopotentials and plane wave basis expansions, typically employ periodic boundary conditions (PBC), and use fast fourier transforms (FFT), to study materials properties. To extend this powerful calculational technique to systems lacking full three-dimensional periodicity - e.g., molecules, surfaces (slabs), or local defects in bulk materials - entails the use of the supercell approximation.3,4 Three dimensional periodicity is artificially imposed by periodically repeating the system in aperiodic directions. The finite supercell contains the immediate region around the system, and an isolated defect is transformed into a periodic array of defects.

Short of the (computationally unachievable) limit of infinitely large supercells, this construction incorporates an error in the simulation of an isolated system as the system interacts with its (artificial) periodic images. ${ }^{5-9}$ The most critical interaction is electrostatic, as the tails of the potentials generated by local electrostatic moments of the defect can be both large and long range. These potential tails overlap supercell boundaries, corrupting the local potential of the "isolated" defect. For supercells separated by vacuum gaps, e.g. molecules ${ }^{7-9}$, or repeated slabs,, 69 it is possible to avoid, rigorously, the error of interacting multipole moments. A singular exception is the treatment of charge, particularly charged defects in extended systems. All of modern semiconductor electronics is based on controlling the release and capture of electrons and holes at defects, and charge states play large roles in diffusion mechanisms and energetics. It is highly desirable, therefore, to be able to perform physically meaningful calculations of the properties of those charged defects from first principles. The difficulty stems from the fact that the electrostatic potentials, and, hence, energetics, of a periodic array of charged supercells diverges.

In this Letter, I report calculations of the ionization potential of the anion vacancy in $\mathrm{NaCl}$, in one-, two-, and three-dimensional periodic geometries. I explicitly demonstrate that the 
conventional approach to avoid Coulombic divergence in charged supercells, neutralizing the net charge using a flat background density, or "jellium", fails to represent the topology of the local electrostatic potential accurately. The inaccuracy in the potential is so large that for materials with modest band gap energies, such as typical semiconductors, the results for charged defects obtained with a jellium-based supercell construction are unreliable. I present an alternate approach, a generalization to extended systems of the local moment countercharge (LMCC) method, ${ }^{9}$ that correctly represents the electrostatic potential within the volume of the supercell, and enables the properties of charge defects in extended systems to be computed with high fidelity.

To understand how a jellium-based Ansatz fails to adequately represent the local electrostatic potential in a charged supercell calculation, consider the simple example of the potential about an isolated Gaussian density distribution, with unit charge, in vacuum. The exact electrostatic potential generated by such a charge distribution can be computed analytically, and compared to the potential generated via the supercell approximation using a flat background density to neutralize the charge. A Gaussian with exponent $0.3 / b_{0 h r^{2}}$ is placed in a cubic supercell with edge length $L$. Figure 1 plots the difference between the jellium-based electrostatic potential computed via an FFT, and the exact potential computed analytically, for a cube with $L=10$ bohr. In reciprocal space, the potential is only known to within a global constant. For this comparison, the constant is chosen to make the analytic and jellium-based potentials equal at the cube center, and the difference computed over the volume of the cell. At the center of a cube face, the exact potential is given by $q / r$. (the Gaussian being contained within the volume of the cube), but the jellium-based calculation gives a potential that is too high by $2.01 \mathrm{eV}$.

The error described here derives from contamination of the local potential by the potential tails of the periodic images of the charge, a problem the jellium compensating charge does address. The prescription of Makov and Payne ${ }^{7}$ describes how to construct an energy properly when jellium is used to neutralize the cell, but this energy correction is built upon an adulterated potential.

For a typical $\mathrm{Si}(\mathrm{Ge})$ 64-atom cubic supercell, $L=20.52$ (21.39) bohr (using experimental lattice parameters). A jellium-neutralized calculation yields a potential error for this supercell of 
$1.00(0.94) \mathrm{eV}$. This error is of the same order as a band gap energy in silicon. For a larger 216atom cubic supercell, this error only reduces to $0.67(0.62) \mathrm{eV}$, the size of a band gap energy in germanium. As the cube edge length $L$ increases, the size of the jellium supercell potential error scales as $1 / L$. Increasing supercell size to reduce the jellium potential error to an acceptable fraction of band gap energies is impractical. The use of a jellium as a compensating charge in supercell calculations of defects in semiconductors is an approximation of convenience, and an approximation that this analysis exposes as inadequate. The jellium compensating charge artificially screens the $q / r$ potential from the charge in the defect. One consequence of this overscreening is that the stability of a charged defect is overestimated, as the potential difference between the defect (supercell center) and the bulk-like (supercell boundary) region is reduced.

If the jellium-based Ansatz fails, how is a physically realistic supercell calculation of a charged defect to be accomplished? Since the difficulty derives from the long range Coulomb tails from the periodic system images, the solution is to eliminate the charge that is the source of those potential tails. For vacuum gap supercells, e.g. for molecules or repeated slab supercells where the system to be studied is separated from its images by vacuum, the LMCC method ${ }^{9}$ provides an alternate solution to Poisson's Equation that exactly removes the spurious effects on the potential (and energy) of electrostatic moments in the supercell. A model density $n_{\mathrm{LM}}(r)$, composed of a Gaussian array of charges $n_{\mathrm{g}}(r)$,

$$
n_{\mathrm{LM}}(r)=\sum_{\mathrm{g}} \mathrm{c}_{\mathrm{g}} n_{\mathrm{g}}\left(r-R_{\mathrm{g}}\right)
$$

is constructed to match the moments of the local system. The total charge distribution of the system $\rho(r)$ is represented in two pieces, this model density and a remainder piece $\rho^{\prime}(r)$ :

$$
\rho(r)=\rho^{\prime}(r)+n_{\mathrm{LM}}(r) .
$$

The remainder charge distribution $\rho^{\prime}(r)$ has no moments, and periodic boundary conditions and FFT's are used to accurately compute the electrostatic potential $\phi_{\mathrm{PBC}}^{\prime}(r)$. The potential $\phi_{\mathrm{LM}}(r)$ from the LMCC is computed treating the Gaussians as isolated, with the potentials cut off at the 
boundary of the supercell. The solution to Poisson's Equation is linear in the density, and the total potential is simply the sum of these potentials:

$$
\phi(r)=\phi_{\mathrm{PBC}}^{\prime}(r)+\phi_{\mathrm{LM}}(r)
$$

This solution does not make errors in the computed potential from artificial images of the moments in the charge distribution, up to the moments contained in the LMCC. The object is to treat an isolated defect or molecule, and the mixing of boundary conditions in the solution of Poisson's Equation is designed to achieve this end, much in the spirit of a Green's function method.

Two issues arise in extending the LMCC to charged defects in bulk systems. First, the potential $\phi_{\mathrm{LM}}(r)$, in general, has discontinuities at the supercell boundaries. For molecules, these discontinuities occur in vacuum, and can be ignored. For bulk defects, the discontinuities in the potential occur in the bulk medium and must be avoided. Second, a localized charge at a bulk defect interacts with the medium outside a volume defined by the supercell. The defects should not interact across supercell boundaries, but the potential from the charge of an isolated defect, in reality, extends outside the volume defined by a local supercell. The LMCC potential is truncated at the supercell boundary. The interaction of the local charge with the extended bulk environment must be added into the calculation.

The first issue is addressed by using as the local supercell, not the parallelepiped defined by the lattice repeat vectors of the supercell, but, rather, the Wigner-Seitz (WS) volume around the position of the local charge, as illustrated in Figure 2. Every boundary is equidistant between charges in neighboring supercells, and the potentials in each local volume are equal at the boundary. All space is tiled with these local WS volumes, regaining full periodic translation symmetry, with potentials that are continuous. The contamination of the local potential by tails of potentials from artificial images is rigorously avoided.

The second issue has two parts. In the $\mathrm{NaCl}$ crystal, the energy of the Madelung interaction of the local vacancy charge with the extended environment needs to be evaluated (outside the volume of the local WS cell). This integral is easily evaluated explicitly. There is also a 
contribution to the energy from the dielectric response of the bulk to the presence of a local charge. This can be empirically estimated using the static dielectric constant of the reference bulk material, in the manner of Leslie and Gillan, 5 or extrapolated from calculations varying the size of the supercell. Extrapolation using first principles results is used here.

Three series of calculations for $\mathrm{NaCl}$ are presented: a one-dimensional single strand of alternating $\mathrm{Na}$ and $\mathrm{Cl}$, a two-dimensional square single sheet of $\mathrm{NaCl}$, and full three-dimensional bulk $\mathrm{NaCl}$. An anion vacancy is created, and the ionization potential (IP) of the $F$ center electron computed as a function of the size of the supercell. I consider only electronic polarization effects; the atoms are held fixed in the bulk equilibrium sites $\left(R_{\mathrm{Cl}-\mathrm{Na}}=4.488\right.$ bohr[1D], 4.95[2D], 5.20[3D]). The calculations use the local density functional approximation ${ }^{2}$ to density functional theory, 1 and are performed using a Gaussian-based linear combination of atomic orbitals method, ${ }^{10}$ with first principles norm conserving pseudopotentials. ${ }^{11}$ The $\mathrm{Na}$ atom is treated as "large-core" ( $2 s$ and $2 p$ shells are considered core electrons) and a non-linear core correction is invoked. ${ }^{12}$ A high-quality "double-zeta plus polarization" basis set is used for all atoms: two radial degrees of freedom for occupied $s$ - and $p$-shells, plus a $d$-shell to allow for angular polarization. Past experience has shown this level of basis reproduces well the results of converged plane wave calculations. ${ }^{13}$

Figures 3-5 present computed ionization potentials (referenced to vacuum) for the $\mathrm{Cl}$ vacancy in $1 \mathrm{D}-, 2 \mathrm{D}$-, and 3D-NaCl, as a function of the size of the supercell (in number of atoms). The $1 \mathrm{D}$ ionization potential shown in Figure 3 is fully converged, to $4.60 \mathrm{eV}$, after only -20 atoms or 10 repeat units. The results are insensitive to the distance between strands, once the strands are far enough apart that they do not overlap, i.e., the strands are separated by vacuum. As the supercell is made longer (the separation between vacancies along a strand increased), the local WS polarization region is larger and the total energy becomes lower. The IP converges approximately as $L^{-3}$, as expected from classic dielectric screening in one dimension. This stands in constrast to the $L^{-1}$ that would result from a jellium-neutralized calculation. 
The 2D ionization potential depicted in Figure 4 converges more slowly. The computed IP for polar $N \times N$ supercells, marked by squares, and non-polar $N \sqrt{2} \times N \sqrt{2}$ supercells, marked by diamonds, fall smoothly along the same curve. Once again, the results are insensitive to the spacing between the sheets once the sheets are separated by vacuum ( $20 \mathrm{bohr})$, and are only dependent on the lateral separation between defects within a sheet. As a function of supercell size, the computed IP energy converges toward an asymptotic limit of $4.36 \mathrm{eV}$. The larger the supercell, the larger the local WS polarization region, and the lower the energy. The 324-atom $(9 \sqrt{2} \times 9 \sqrt{2})$ calculation is within $0.02 \mathrm{eV}$ of this limit, but a 50 -atom $(5 \times 5)$ supercell is already within $\sim 0.1 \mathrm{eV}$. The observed $L^{-2}$ scaling of the IP is exactly that expected from classic dielectric screening in two dimensions.

The IP calculations for bulk $\mathrm{NaCl}$ are also done using both non-polar (cubic) supercells, and polar $(f c c)$ supercells, marked with squares and diamonds, respectively. With full threedimensional screening, the computed IP converges much more slowly. The results from the polar and non-polar supercells fall along the same curve. The convergence of the IP in three dimensions scales as $1 / L$, again as expected from classic dielectric screening. It is important to note that this convergence with supercell size is due entirely to the screening energy from electronic polarization within the changing WS volume, in response to the charged vacancy. The IP variation is not a failure to include a self-energy term, as described in Makov and Payne ${ }^{7}$ for a jellium-based neutralization, nor, as described above, a failure to represent the local potential properly. Without the artificial screening of the charge given by a jellium supercell potential, the true magnitude of the electronic polarization to a point charge is revealed.

The largest bulk supercell considered here is a 250-atom fcc cell, and the IP for a $\mathrm{Cl}$ vacancy in that cell is computed to be $3.69 \mathrm{eV}$. The asymptotic limit, from both the fcc and cubic cell series, is marked by the dotted line in Figure 5 and is almost $0.5 \mathrm{eV}$ lower. The 64-atom cubic supercell, which usually might be deemed sufficiently large for defect calculations, misses almost $0.8 \mathrm{eV}$ in polarization energy, compared to the asymptotic limit. For computation of total energetics, it is crucial that this polarization be properly included. For $\mathrm{NaCl}$, this energy may not 
be significant, but, again, in semiconductors, this energy is comparable to a band gap energy. The large screening term is the reason why the ionization potential in the $3 \mathrm{D}$ system $(3.2 \mathrm{eV})$ is so much smaller than in the $1 \mathrm{D}(4.60 \mathrm{eV})$ and $2 \mathrm{D}(4.36 \mathrm{eV})$ systems, despite having a larger Madelung potential binding the electron.

For defects in a single material, such as silicon, this polarization term need be only calculated

once. It can either be explicitly extrapolated, as above, for one charged defect, or estimated empirically using the static dielectric constant, and used for all defect calculations with the same charge in the same supercell. For different defects in the same supercell, the polarization energy will be identical, and depends solely on the charge on the defect.

In summary, I have presented first principle calculations of total energetics of charged vacancies in $\mathrm{NaCl}$ that accurately embody the correct electrostatic interactions appropriate to isolated defects. I have demonstrated that the standard recipe for studying charged defects in bulk systems, using jellium as a neutralizing agent in supercell calculations, incorporates an error in the computed electrostatic potential that is comparable to the energy scale of physical interest. The generalized LMCC approach I present eliminates the unphysical interaction of charges between supercells and enables the computation of the properties of charged defects in bulk systems using the correct local electrostatic potential.

\section{Acknowledgments}

Sandia is a multiprogram laboratory operated by Sandia Corporation, a Lockheed Martin Company, for the United States Department of Energy, under Contract No. DEAC0494AL85000.

\section{References}

1 P. Hohenberg and W. Kohn, Phys. Rev. 136, B864 (1964).

2 W. Kohn and L.J. Sham, Phys. Rev. 140, A1133 (1965); see also Theory of the Inhomogeneous Electron Gas, edited by S. Lundqvist and N.M. March (Plenum, New York, 1983). 
${ }^{3}$ R.P. Messmer, B. McCarroll and C.M. Singal, J. Vac. Sci. Technol. 9, 891 (1972);

G.D. Watkins and R.P. Messmer, in Computational Methods for Large Molecules and Localized States in Solids, edited by F. Herman, A.D. McLean, and R.K. Nesbet, Plenum Press (New York, 1973) p. 133; R.P. Messmer and G.D. Watkins, in Radiation Damage and Defects in Semiconductors, Conference Series No. 16, The Institute of Physics, (London, 1973), p. 255.

${ }^{4}$ M.L. Cohen, M. Schlüter, J.R. Chelikowsky, and S.G. Louie, Phys. Rev. B 12, 5575 (1975).

5 M. Leslie and M.J. Gillan, J. Phys. C 18, 973 (1985).

6 J. Neugebauer and M. Scheffler, Phys. Rev. B 46, 16067 (1992).

7 G. Makov and M.C. Payne, Phys. Rev. B 51, 4014 (1995).

8 M.R. Jarvis, I.D. White, R.W. Godby, and M.C. Payne, Phys. Rev. B 56, 14972 (1997).

9 P.A. Schultz, Phys. Rev. B (in press).

10 P.A. Schultz and P.J. Feibelman, SeqQuest Program, unpublished; for a description of the method see: P.J. Feibelman, Phys. Rev. B 35, 2626 (1987).

11 D.R. Hamann, Phys. Rev. B 40, 2980 (1989).

12 S.G. Louie, S. Froyen, and M.L. Cohen, Phys. Rev. B 26, 1738 (1982).

13 J.S. Nelson, E.B. Stechel, A.F. Wright, S.J. Plimpton, P.A. Schultz, and M.P. Sears, Phys. Rev. B 52, 9354 (1995). 


\section{Figure Captions.}

Figure 1. Error in the computed electrostatic potential in a supercell calculation using jellium as the neutralizing agent. The exact potential is computed analytically for a Gaussian density distribution (decay constant $=0.3 \mathrm{bohr}^{-2}$ ) in a cubic volume (edge $=10 \mathrm{bohr}$ ), and the difference with the potential computed using an FFT after neutralization by jellium. The difference is plotted along a line connecting the centers of opposite faces and going through the center of the cell, as shown in the inset. The error at the face center is $2.01 \mathrm{eV}$.

Figure 2. Schematic of method to construct local supercell volume for viable LMCC construction in extended systems. To prevent discontinuities in the potential in bulk, the local volume must be defined using Wigner-Seitz cells around the local charge, rather than a parallelepiped defined by the supercell repeat vectors. The full translational symmetry of bulk is retained as the local potential from the LMCC is periodically repeated over all space.

Figure 3. The computed ionization potential for a chlorine vacancy in a $1 \mathrm{D} \mathrm{NaCl}$ chain, as a function of the size of the supercell.

Figure 4. The computed ionization potential for a chlorine vacancy in a $2 \mathrm{D}$ square $\mathrm{NaCl}$ sheet, as a function of the size of supercell. Points from polar $N \times N$ cells (inset: smaller $1 \times 1$ two atom cell) are plotted with squares, and non-polar $N \sqrt{2} \times N \sqrt{2}$ cells (inset: larger rotated four-atom $\sqrt{2} \times \sqrt{2}$ cell) are plotted with diamonds.

Figure 5. The computed ionization potential for a chlorine vacancy in bulk $\mathrm{NaCl}$, as a function of the size of supercell. Points from non-polar simple cubic cells are plotted with squares, while points from polar fcc supercells are plotted with diamonds. The dashed line denotes the asymptotic limit of the IP, extrapolated from the points shown. 
11

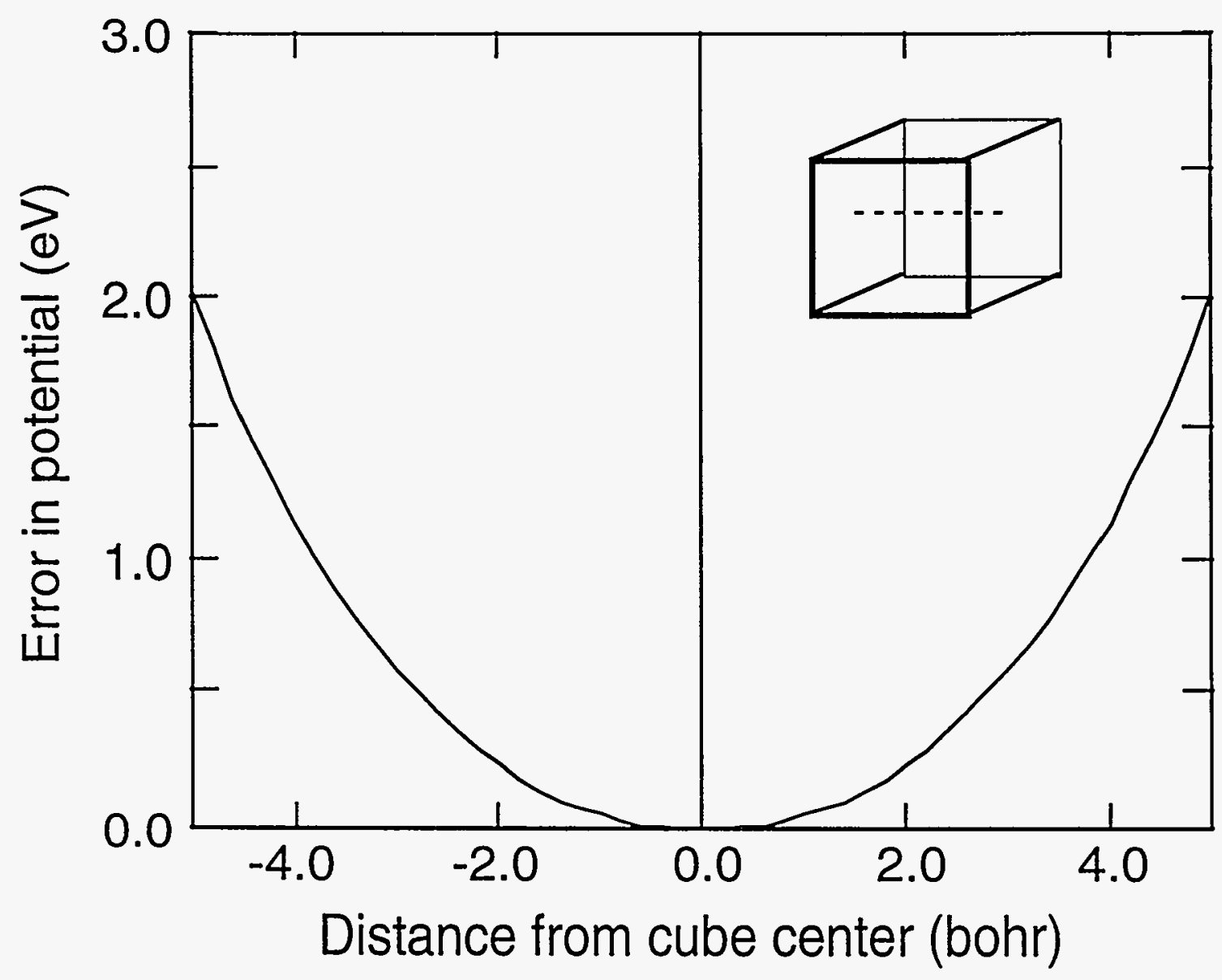

Figure 1 - Schultz 


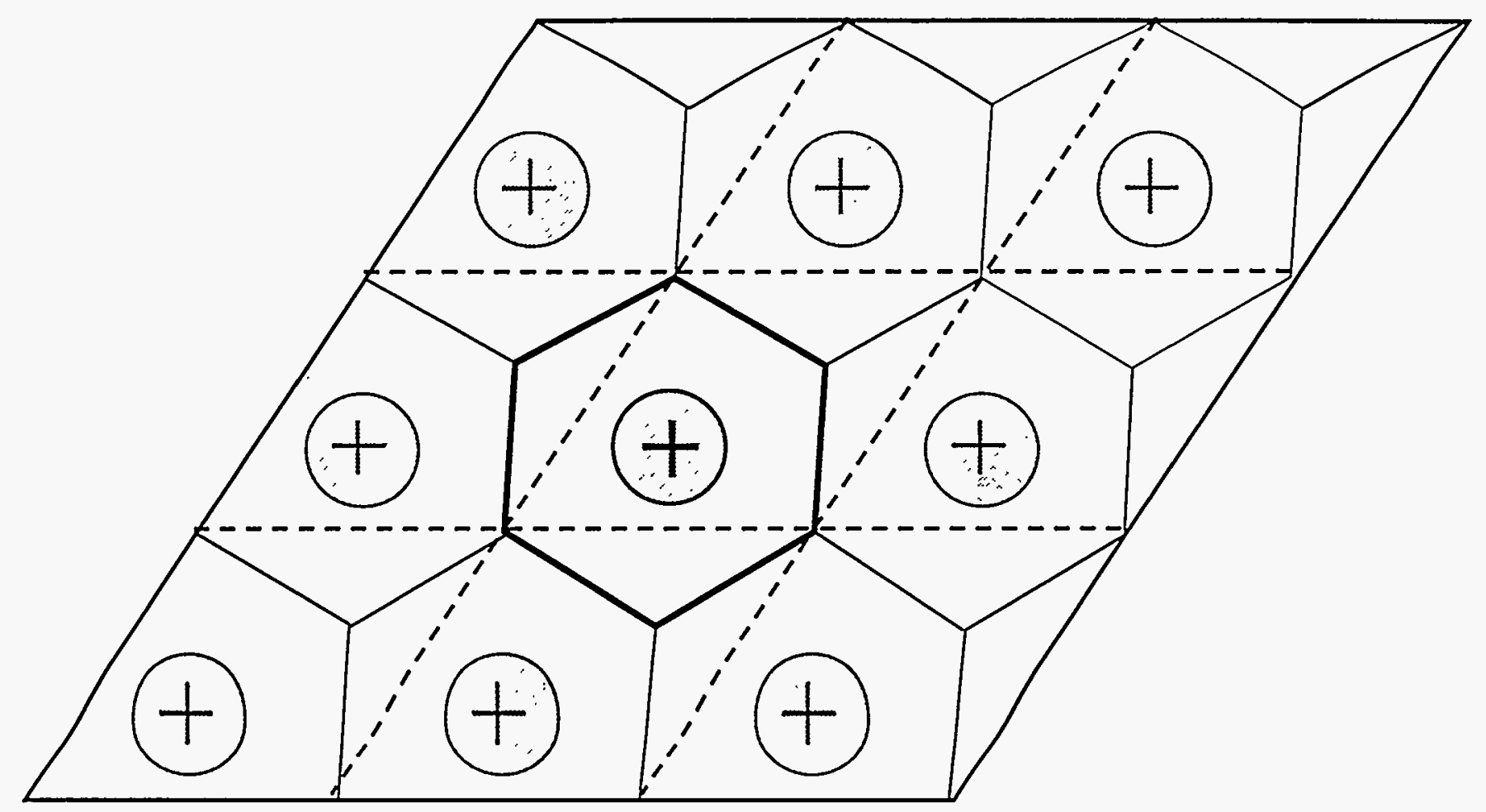

Figure 2 - Schultz 


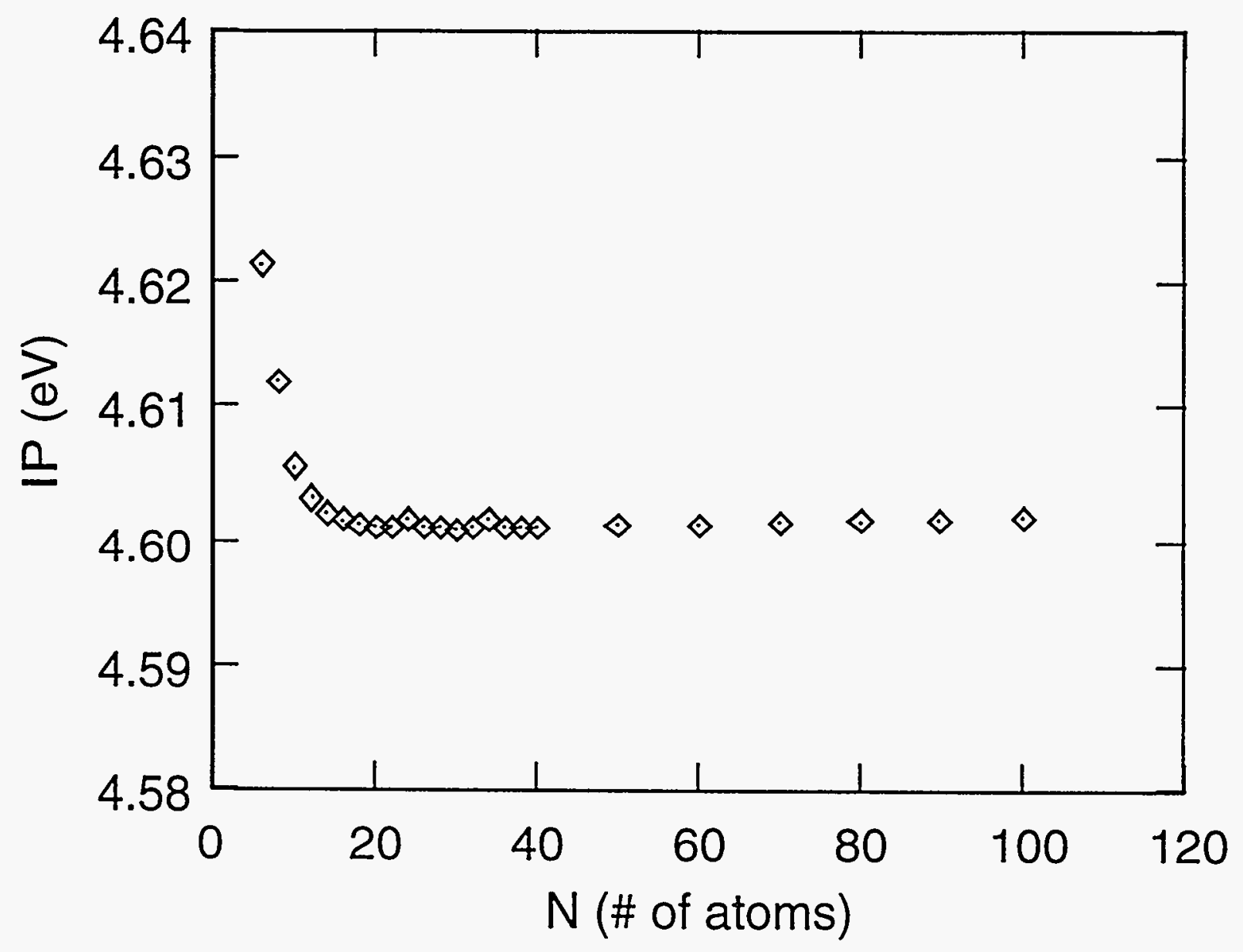

Figure 3 - Schultz 


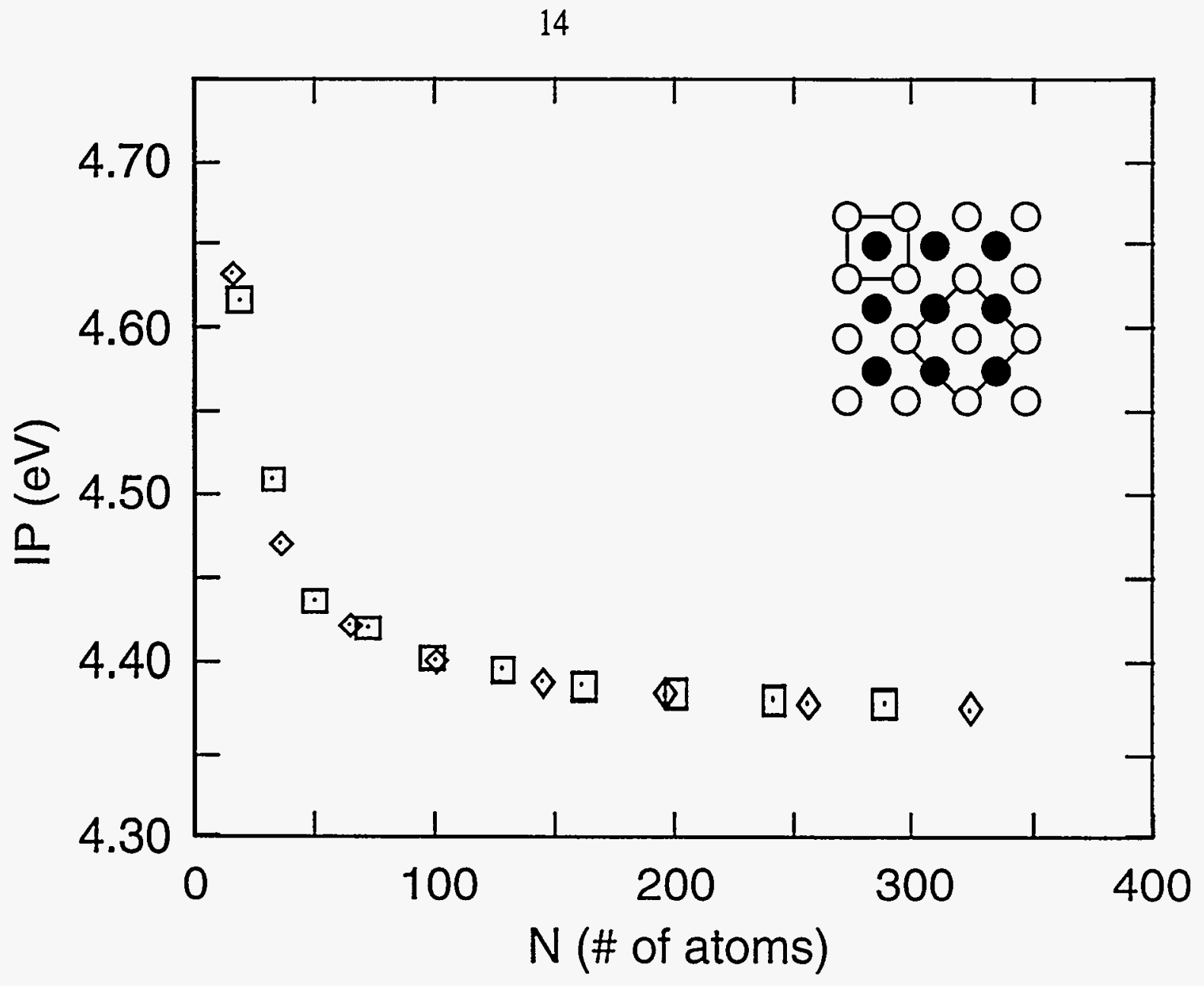

Figure 4 - Schultz 
15

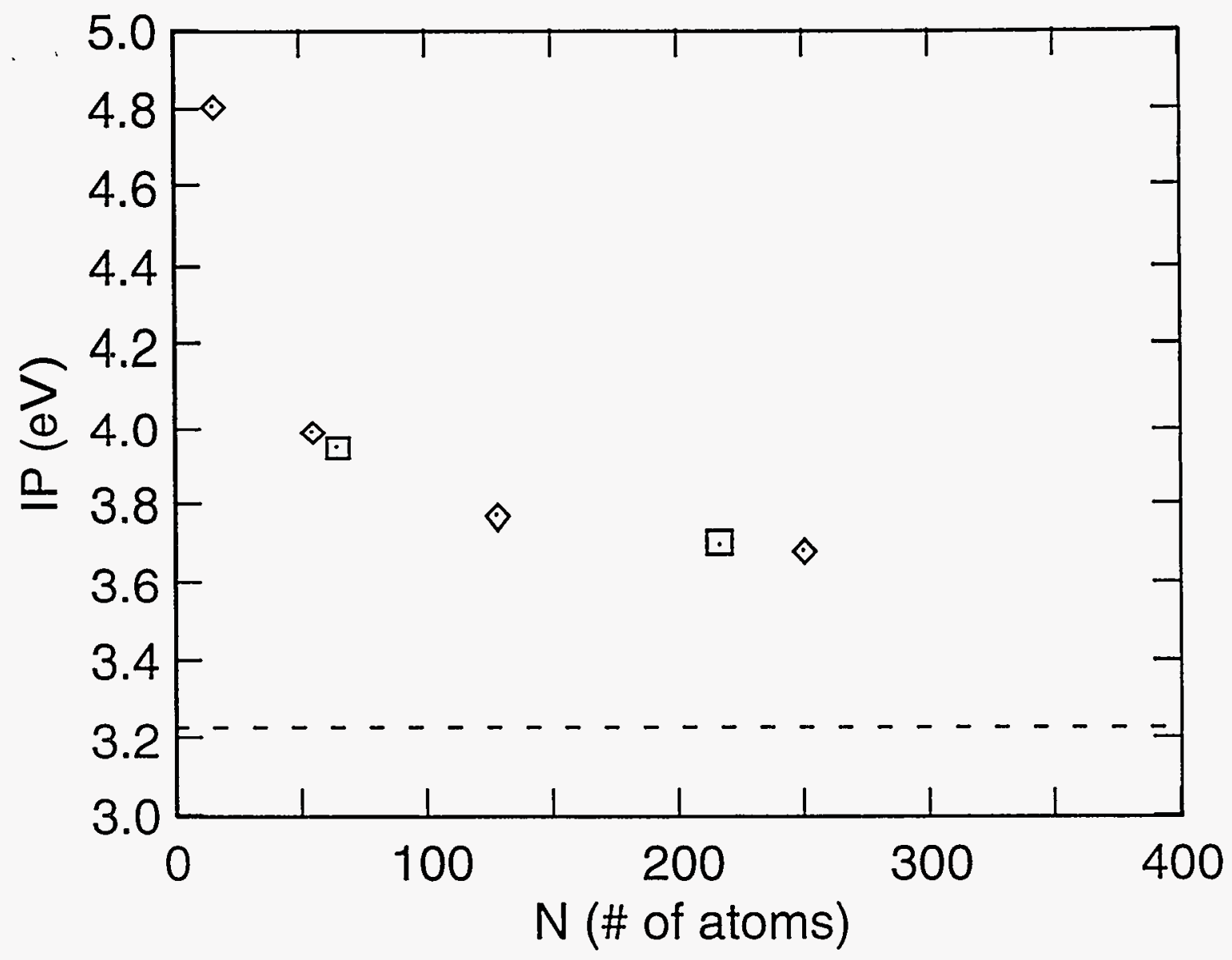

Figure 5 - Schultz 